\title{
THE
}

\section{Function of the Heterocercal Tail in Sharks: Quantitative Wake Dynamics During Steady Horizontal Swimming and Vertical Maneuvering}

C. D. Wilga

University of Rhode Island, cwilga@uri.edu

G. V. Lauder

Follow this and additional works at: https://digitalcommons.uri.edu/bio_facpubs

Creative Commons License

c) (7) (8)

This work is licensed under a Creative Commons Attribution-Noncommercial 3.0 License

\section{Citation/Publisher Attribution}

Wilga, C. D. and G. V. Lauder. "Function of the Heterocercal Tail in Sharks: Quantitative Wake Dynamics During Steady Horizontal Swimming and Vertical Maneuvering." Journal of Experimental Biology. 205(16):2365-2374. August 2002.

Available at http://jeb.biologists.org/content/205/16/2365.full.pdf+html JEB4298E

This Article is brought to you for free and open access by the Biological Sciences at DigitalCommons@URI. It has been accepted for inclusion in Biological Sciences Faculty Publications by an authorized administrator of DigitalCommons@URI. For more information, please contact digitalcommons-group@uri.edu. 


\title{
Function of the heterocercal tail in sharks: quantitative wake dynamics during steady horizontal swimming and vertical maneuvering
}

\author{
C. D. Wilga ${ }^{1, *}$ and G. V. Lauder ${ }^{2}$ \\ ${ }^{1}$ Department of Biological Sciences, University of Rhode Island, 100 Flagg Road, Kingston, RI 02881, USA and \\ ${ }^{2}$ Museum of Comparative Zoology, Harvard University, Cambridge, MA 02138, USA \\ *e-mail: cwilga@uri.edu \\ Accepted 25 May 2002
}

\begin{abstract}
Summary
The function of the heterocercal tail in sharks has long been debated in the literature. Previous kinematic data have supported the classical theory which proposes that the beating of the heterocercal caudal fin during steady horizontal locomotion pushes posteroventrally on the water, generating a reactive force directed anterodorsally and causing rotation around the center of mass. An alternative model suggests that the heterocercal shark tail functions to direct reaction forces through the center of mass. In this paper, we quantify the function of the tail in two species of shark and compare shark tail function with previous hydrodynamic data on the heterocercal tail of sturgeon Acipenser transmontanus. To address the two models of shark heterocercal tail function, we applied the technique of digital particle image velocimetry (DPIV) to quantify the wake of two species of shark swimming in a flow tank. Both steady horizontal locomotion and vertical maneuvering were analyzed. We used DPIV with both horizontal and vertical light sheet orientations to quantify patterns of wake velocity and vorticity behind the heterocercal tail of leopard sharks (Triakis semifasciata) and bamboo sharks (Chiloscyllium punctatum) swimming at $1.0 L^{-1}$, where $L$ is total body length. Two synchronized high-speed video cameras allowed simultaneous measurement of shark body position and wake structure. We measured the orientation of tail vortices shed into the wake and the orientation of the central jet through the core of these vortices relative to body orientation. Analysis of flow geometry indicates that

the tail of both leopard and bamboo shark generates strongly tilted vortex rings with a mean jet angle of approximately $30^{\circ}$ below horizontal during steady horizontal swimming. The corresponding angle of the reaction force is much greater than body angle (mean $11^{\circ}$ ) and the angle of the path of motion of the center of mass (mean approximately $0^{\circ}$ ), thus strongly supporting the classical model of heterocercal tail function for steady horizontal locomotion. Vortex jet angle varies significantly with body angle changes during vertical maneuvering, but sharks show no evidence of active reorientation of jet angle relative to body angle, as was seen in a previous study on the function of sturgeon tail. Vortex jet orientation is significantly more inclined than the relatively horizontal jet generated by sturgeon tail vortex rings, demonstrating substantial differences in function in the heterocercal tails of sharks and sturgeon.

We present a summary of forces on a swimming shark integrating data obtained here on the tail with previous data on pectoral fin and body function. Body orientation plays a critical role in the overall force balance and compensates for torques generated by the tail. The pectoral fins do not generate lift during steady horizontal locomotion, but play an important hydrodynamic role during vertical maneuvering.

Key words: swimming, heterocercal tail, flow visualization, hydrodynamics, digital particle image velocimetry, shark, Triakis semifasciata, Chiloscyllium punctatum.
\end{abstract}

\section{Introduction}

Two competing models have been presented to explain how the heterocercal tail functions during locomotion in sharks. First, the classical model of locomotion in sharks proposes that the heterocercal tail functions by transmitting posteroventral momentum to the water during steady horizontal swimming, thereby producing an anterodorsal reaction force (Alexander, 1965; Ferry and Lauder, 1996; Lauder, 2000). Since this reaction force is directed above the center of mass, it produces a torque around the center of mass that must be counteracted by lift forces generated at the anterior end of the body. According to the classical model, the pectoral fins are thought to be upwardly inclined and are believed to generate the lift forces countering the torque produced by the heterocercal tail in order to achieve rotational equilibrium. Wilga and Lauder (2000, 2001) have shown experimentally that the pectoral fins of two species of shark do not in fact generate lift forces during steady horizontal locomotion, although they do play an active hydrodynamic role during maneuvering. Using the 

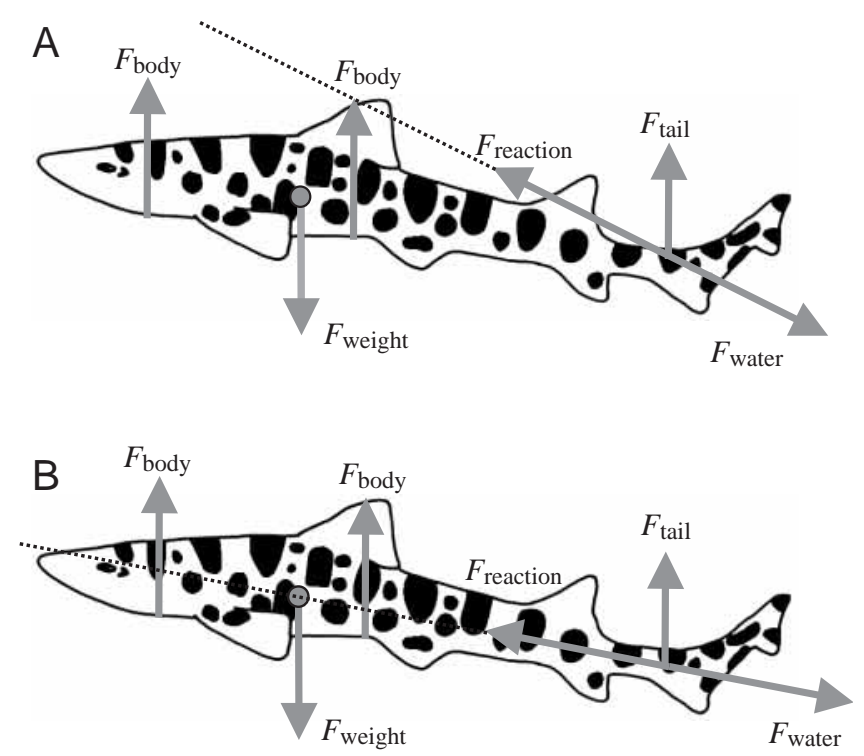

Fig. 1. Schematic summary of two alternative models illustrating the forces acting on the body of a shark during steady horizontal swimming. (A) Modified version of the classical model (with data on body angle and pectoral fin function incorporated from Wilga and Lauder, 2000, 2001) in which the beating of the tail is proposed to generate an upward lift force $\left(F_{\text {tail }}\right)$ that generates a torque around the center of mass (shaded circle). Force on the water is directed posteroventrally $\left(F_{\text {water }}\right)$, and an equal and opposite reaction force is directed anterodorsally, dorsal to the center of mass $\left(F_{\text {reaction}}\right)$. Torques generated by the tail are countered by equal and opposite torques resulting from lift forces produced by the body $\left(F_{\text {body }}\right)$, which has a positive angle of attack during horizontal locomotion. The net upward lift forces are balanced by the weight ( $\left.F_{\text {weight }}\right)$ of the negatively buoyant shark. The pectoral fins do not generate lift during steady horizontal locomotion (Wilga and Lauder, 2000, 2001) and, hence, no forces are shown acting on these fins. (B) Modified version of the model of Thomson (1976) (to include our previously published data on shark body angle and pectoral fin function) in which the tail generates a reaction force that is directed anteriorly through the center of mass.

experimental hydrodynamic technique of digital particle image velocimetry (DPIV), Wilga and Lauder (2000, 2001) showed that leopard and bamboo sharks balance rotational moments during steady horizontal locomotion by altering the angle of the body to the incident flow and not by generating lift with the pectoral fins. Body angle is also used to generate lift forces anteriorly which, summed with lift generated by the tail, are equal and opposite to the weight of the shark in the water. This modified classical view of shark locomotion is summarized in Fig. 1A.

The second view of heterocercal tail function in sharks was proposed by Thomson (1976; see also Thomson and Simenak, 1977). In this model (summarized in Fig. 1B), the shark tail generates a reaction force directed through the center of mass. No torque is generated by the action of the tail and, hence, no counterbalancing forces need to be generated by the pectoral fins and body.

No experimental hydrodynamic data currently exist to permit a quantitative assessment of the function of the heterocercal tail in sharks during in vivo locomotion. Some progress in understanding shark tail function has been made using manipulative studies of isolated tails or tail models (Grove and Newell, 1936; Affleck, 1950; Alexander, 1965; Simons, 1970). The three-dimensional kinematic study of freely swimming sharks of Ferry and Lauder (1996) and the dye-stream tracking in their study strongly supported the classical model, while the drawings of tail position during swimming by Thomson (1976) supported the alternative model.

To quantify the function of the heterocercal tail in sharks and resolve the two alternative views discussed above, it is necessary to evaluate the forces generated by the tail during both steady horizontal locomotion and vertical maneuvering. The technique of DPIV has been used successfully to analyze the hydrodynamic function of pectoral fins in both sharks and sturgeon Acipenser transmontanus (Wilga and Lauder, 1999, 2000,2001 ) and to examine the function of the caudal fin of sturgeon (Liao and Lauder, 2000). DPIV has the advantages of (i) allowing freely swimming animals to be studied in a controlled laboratory setting, (ii) providing detailed quantitative data on water flow in the wake of swimming fishes (see Drucker and Lauder, 1999, 2000, 2001; Lauder, 2000; Nauen and Lauder, 2001) and (iii) allowing the direction of force application by the tail to the water, and hence the direction of the reaction force, to be calculated.

In this study, we use the technique of DPIV to address several questions. First, does the heterocercal tail in sharks swimming horizontally generate a jet flow that is oriented at a large posteroventral angle, as predicted by the classical model (Ferry and Lauder, 1996), or is the tail vortex jet flow oriented so as to produce reaction forces directed through the center of mass, as predicted by Thomson (1976)? Second, does the hydrodynamic function of the shark tail change during vertical maneuvering? Third, do sharks adjust vortex jet angle relative to their path of motion when maneuvering vertically? Fourth, are tail hydrodynamics in sharks comparable with that of the similarly shaped heterocercal tail in sturgeon, which can alter jet angle relative to the path of motion of the body (Liao and Lauder, 2000)? We address these questions using leopard sharks Triakis semifasciata, an epibenthic species, as well as bamboo sharks Chiloscyllium punctatum, a benthic species. These two species differ somewhat in heterocercal tail morphology, allowing us to test the classical model with a moderate diversity of shark tail shapes.

\section{Materials and methods Animals}

Three leopard sharks, Triakis semifasciata Girard, 1854 (21-26 cm total length, $L$ ), were obtained from a commercial fish collector in California (Sea Dwelling Creatures). Three banded bamboo sharks, Chiloscyllium punctatum Bennett, $1830(17-27 \mathrm{~cm} \mathrm{~L})$, were obtained from a wholesale fish distributor. Bamboo and leopard sharks were housed in 13601 aquaria at $25 \pm 1$ and $20 \pm 1^{\circ} \mathrm{C}$, respectively, and maintained on 
a diet of smelt (Osmeridae). Experiments were conducted in a calibrated flow tank as in previous experiments (e.g. Gibb et al., 1994; Jayne and Lauder, 1995; Wilga and Lauder, 1999; Drucker and Lauder, 2001) maintained at the housing temperatures stated above.

\section{Digital particle image velocimetry with simultaneous high- speed recording}

Water flow in the wake of the caudal fin of sharks during steady horizontal swimming and during vertical maneuvering was analyzed using digital particle image velocimetry (DPIV) as in previous research (e.g. Drucker and Lauder, 1999, 2001; Lauder, 2000; Liao and Lauder, 2000; Wilga and Lauder, 2000, 2001). Briefly, water in the flow tank was seeded with $6 \mathrm{~g}$ of near-neutrally buoyant $12 \mu \mathrm{m}$ diameter silver-coated hollow glass beads (density $1.3 \mathrm{~g} \mathrm{~cm}^{-3}$; Potters Industries Inc.). A Coherent $5 \mathrm{~W}$ argon-ion laser was focused into a $1-2 \mathrm{~mm}$ thick by $10 \mathrm{~cm}$ wide light sheet and oriented into vertical and horizontal configurations in separate experiments using mirrors. Particle movement in the water flow was visualized as light reflected by the beads and recorded using a NAC HSV $500 c^{3}$ two-camera synchronized high-speed video system at 250 frames s$^{-1}$ (downloaded image resolution $640 \times 480$ pixels for each camera). The working area of the flow tank was $82 \mathrm{~cm}$ long by $28 \mathrm{~cm}$ wide by $28 \mathrm{~cm}$ high. Water flow and particle reflections in the wake of the caudal fin in lateral (parasagittal) view were recorded by placing one camera perpendicular to the side of the flow tank (Fig. 2). The position of the shark relative to the laser light sheet in lateral view was recorded by a second (synchronized) camera aimed at the swimming shark and slightly overlapping the laser sheet (Fig. 2). This method allowed us to visualize fluid flow and vortex rings shed by the tail while simultaneously recording the orientation and behavior of the swimming shark. This combination proved critical in accurately assessing caudal fin function relative to body angle and in determining the orientation of the reaction force relative to the center of mass.

Leopard sharks, Triakis semifasciata, and bamboo sharks, Chiloscyllium punctatum, were filmed while holding position (steady horizontal swimming) in the flow tank at $1.0 \mathrm{~L} \mathrm{~s}^{-1}$. Five different sequences for each of three individuals for each species were digitized, giving a total of 30 sequences. Rising and sinking (vertical maneuvering) locomotion in the water column were also studied to investigate whether the locomotor function of the tail is to change vertical position. Only leopard sharks were filmed during rising or sinking, and five different sequences for each of three individuals for each behavior were digitized, giving a total of 30 sequences. In total, 300 images were digitized for these measurements of body and caudal fin position during swimming: five fields equally spaced throughout a tailbeat for five tailbeats in four individuals for three behaviors. The vertical laser light sheet was positioned in the center of the tank for all experimental protocols to minimize potential boundary effects from the tank walls on the flow around the fish. Thus, all sequences in which the tail intersected the laser sheet occurred well away from the sides of the flow tank.

We define holding position as the fish maintaining a stationary (within $2 \% \mathrm{Ls}^{-1}$ deviation from a fixed reference point) horizontal (anteroposterior) and vertical position in the water column. Rising and sinking are defined as maintaining horizontal position in the water column while actively increasing or decreasing vertical position by at least $4 \mathrm{~cm} \mathrm{~s}^{-1}$ with minimal lateral deviation. These criteria follow previous studies (Wilga and Lauder, 1999, 2000). We analyzed only those video sequences in which sharks maintained horizontal and vertical position during holding or ascended or descended with near-constant velocity in the water column (in all cases with minimal lateral, upstream-downstream pitching, except when initiating changes in vertical position or roll motions). The initiation of rising and sinking behaviors necessarily
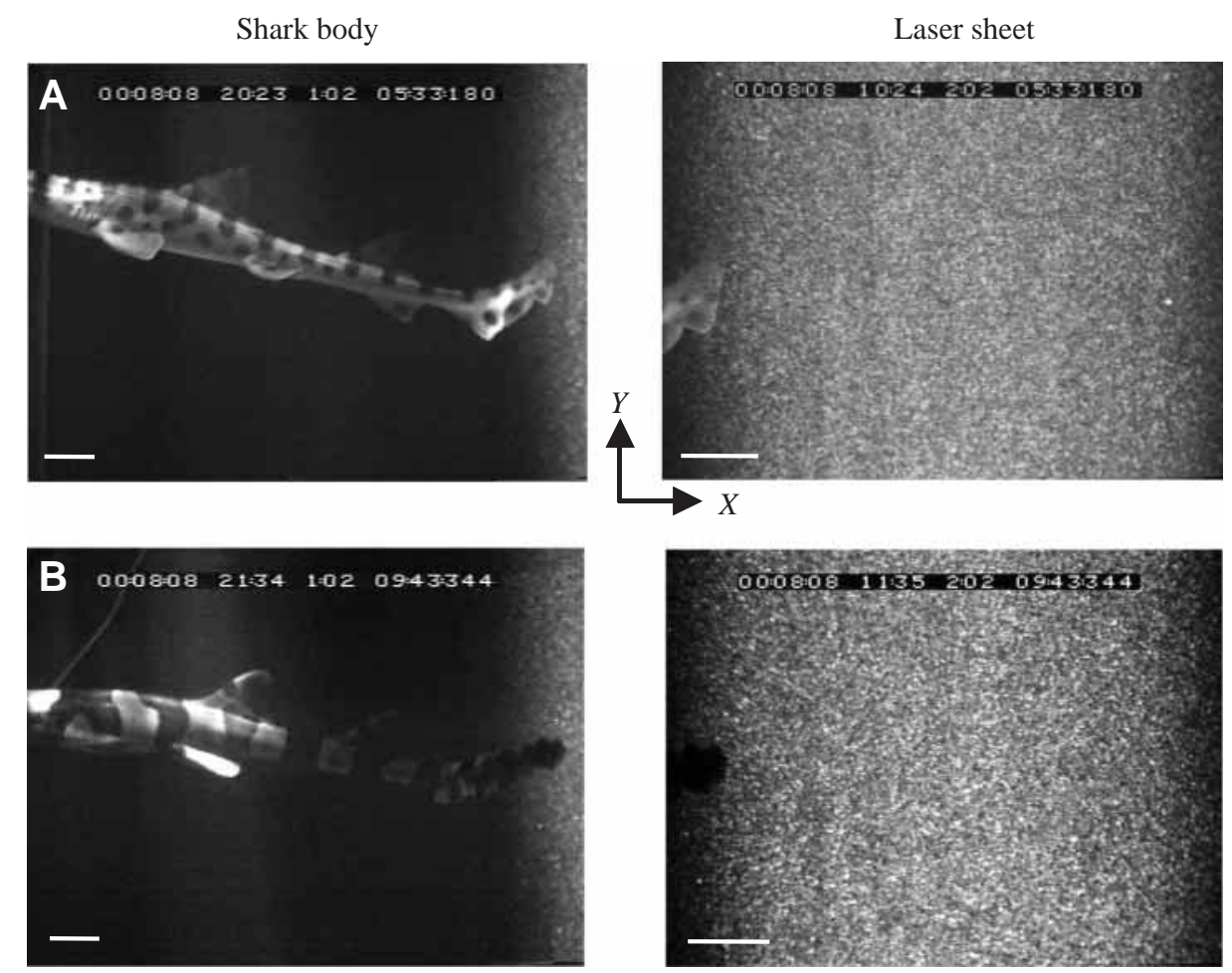

Fig. 2. Synchronized video images illustrating lateral views of (A) Triakis semifasciata (top) and (B) Chiloscyllium punctatum (bottom) during steady horizontal swimming to show body angle and position relative to the edge of the laser sheet (left) and the vertical laser sheet with tail and particles (right). Scale bars, $2 \mathrm{~cm}$. The $X$ and $Y$ axes are marked. 


\section{C. D. Wilga and G. V. Lauder}

Fig. 3. Schematic summary illustrating body and wake variables measured relative to the horizontal: body angle, from a line drawn along the ventral body surface; path of motion of the center of mass; tail angle between the caudal peduncle and dorsal tail lobe; ring axis angle from a line extending between the two centers of vorticity; and mean vortex jet angle. Angle measurements from the variables of interest (dotted lines) to the horizontal (dashed line) are indicated by the curved solid lines. Angles above the horizontal are considered positive and those below the horizontal negative. Ring axis angle was measured from 0 to $180^{\circ}$.

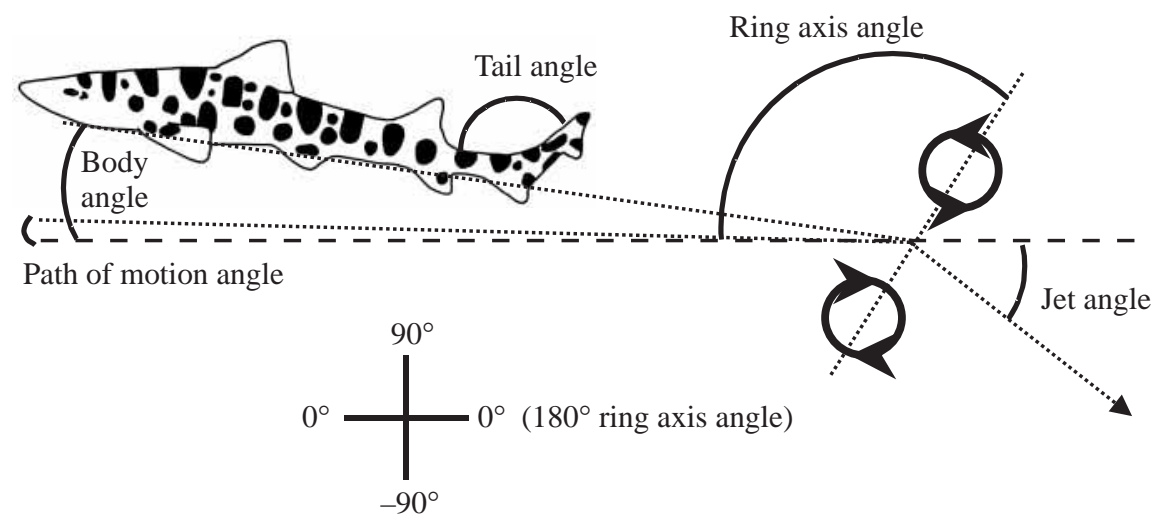

involves pitching movements, as described previously (Wilga and Lauder, 1999, 2000).

Several variables were used to quantify body and tail kinematics for swimming sharks during all behaviors (Fig. 3). Vertical velocity was calculated by digitizing a fixed point (the center of mass) at two points in time. Body angle was measured as the angle between the horizontal and a line drawn along the ventral surface of the body between the anterior base of the pectoral and pelvic fins. Tail angle was measured as the angle between a line representing the dorsal surface of the caudal peduncle and a line indicating the leading edge of the tail (Fig. 3). The path of motion was calculated as the angle between the horizontal and a line connecting a fixed point (the center of mass) at two moments in time (200 ms apart).

Sequences of particle images during station-holding, rising and sinking in the water column during locomotion in sharks were identified using the criteria described above for fin kinematics. Consecutive pairs of video images (4 ms apart) of water flow just downstream of the caudal fin were digitized and analyzed using two-frame cross correlation to produce a $20 \times 20$ matrix of 400 velocity vectors, as for conventional DPIV methods used previously (e.g. Raffel et al., 1998; Drucker and Lauder, 1999, 2000, 2001; Wilga and Lauder, 1999, 2000; Lauder, 2000). In total, 60 image pairs were analyzed using DPIV: five occurrences of pelagic station-

Fig. 4. DPIV analysis of the wake of the tail of a representative Triakis semifasciata (A) and Chiloscyllium punctatum (B) during steady horizontal locomotion. On the left is a tracing of the tail depicting its position relative to single the shed vortex ring visible in this vertical section of the wake. The color plot to the right shows fluid vorticity, with superimposed black velocity vectors representing the results of DPIV calculations based on particle displacements. A strong jet, indicated by the larger velocity vectors, passes between two counter-rotating vortices representing a slice through the vortex ring shed from the tail at the end of each beat. The white dashed line indicates the ring axis cycle. Note that a green color indicates no fluid rotation, a blue color reflects clockwise fluid rotation and a red/yellow color indicates counterclockwise fluid rotation. To assist in visualizing jet flow, a mean horizontal flow of $U=19$ and $U=24 \mathrm{~cm} \mathrm{~s}^{-1}$ was subtracted from each vector for $T$. semifasciata and C. punctatum, respectively. holding from each of three leopard and three bamboo sharks, and five occurrences each of rising and sinking in the water column holding from three leopard sharks.

Fluid flow patterns in the wake of the caudal fin were documented by estimating flow structure using the magnitude and direction of velocity vectors from plots of the $20 \times 20$ matrix of velocity vectors. Mean downstream flow was subtracted from the matrix of velocity vectors to reveal fluid structures in the wake. Fluid vorticity was calculated to
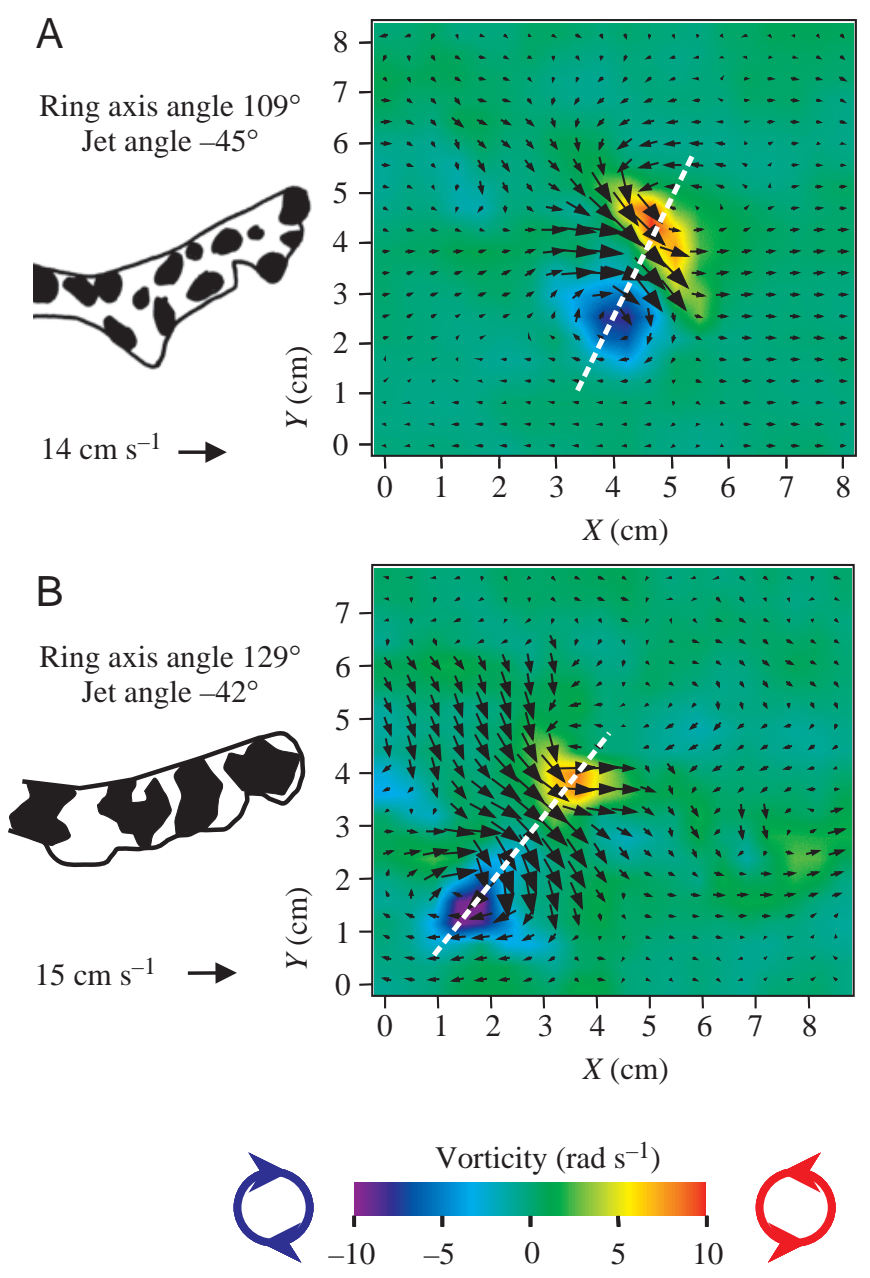
quantify rotational motion in the wake using the velocity vector matrix. Plots of vorticity (e.g. Fig. 4) are shown in order to visualize rotational fluid motion; in these plots, a greenish color indicates low vorticity, a red/orange color is used for counterclockwise fluid movement and a purple/blue color for clockwise motion (Drucker and Lauder, 1999; Wilga and Lauder, 1999, 2000, 2001). Jet angle was calculated by taking the mean angle of 10 high-velocity vectors located in the center of the vortex ring. Ring axis angle was calculated as the angle between the horizontal and a line connecting the centers of the two counter-rotating vortices of the vortex ring (Fig. 3). Ring axis angle was measured directly from the DPIV-analyzed images of the laser light sheet. These conventions correspond to those used by Liao and Lauder (2000) in their study of sturgeon tail function, and the use of those conventions here permits comparison with the sturgeon data.

\section{Statistical analyses}

Mean values of variables measured for each locomotor behavior are reported in Table 1 . These data reflect the means from our a priori categorization of locomotor behavior into holding position, rising or sinking in the water column based on the analysis of the lateral whole-body video sequences. However, because there was extensive variation among sequences in the rapidity of vertical maneuvering and also modest variation in the body angle used during holding position, we also treat the data as continuous without any attempt to categorize individual sequences. In Table 2, we

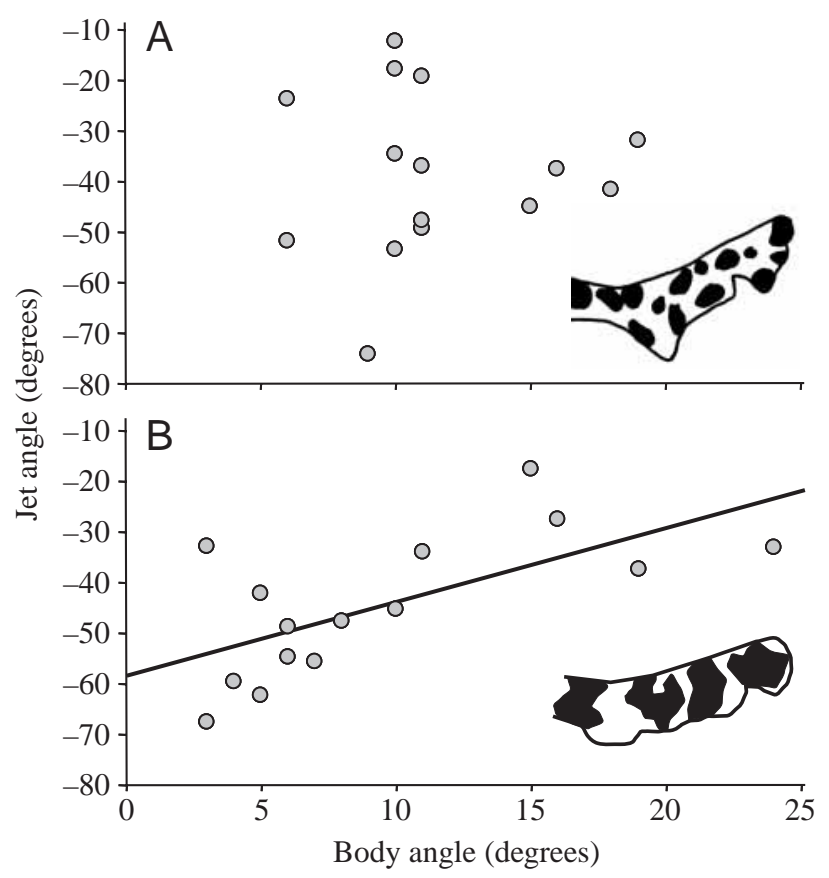

Fig. 5. Plots of jet angle versus body angle in (A) Triakis semifasciata and (B) Chiloscyllium punctatum for steady horizontal locomotion (holding position) only. The solid line for $C$. punctatum indicates a significant linear regression $(y=-58.287+1.452 x$; $P=0.008, r^{2}=0.432$ ). present the means predicted from regression analyses for each variable; these data take into account the entire range of natural variation without $a$ priori categorization and are thus the means used in the Discussion and in the presentation of our overall model of shark locomotor dynamics in Fig. 9. Presentation of both analyses allows comparison with previous analyses of sturgeon locomotor hydrodynamics (Liao and Lauder, 2000), which used the a priori categorization analysis.

Model I least-squares linear regressions with adjusted $r^{2}$ values were calculated using body angle, tail angle, path angle,
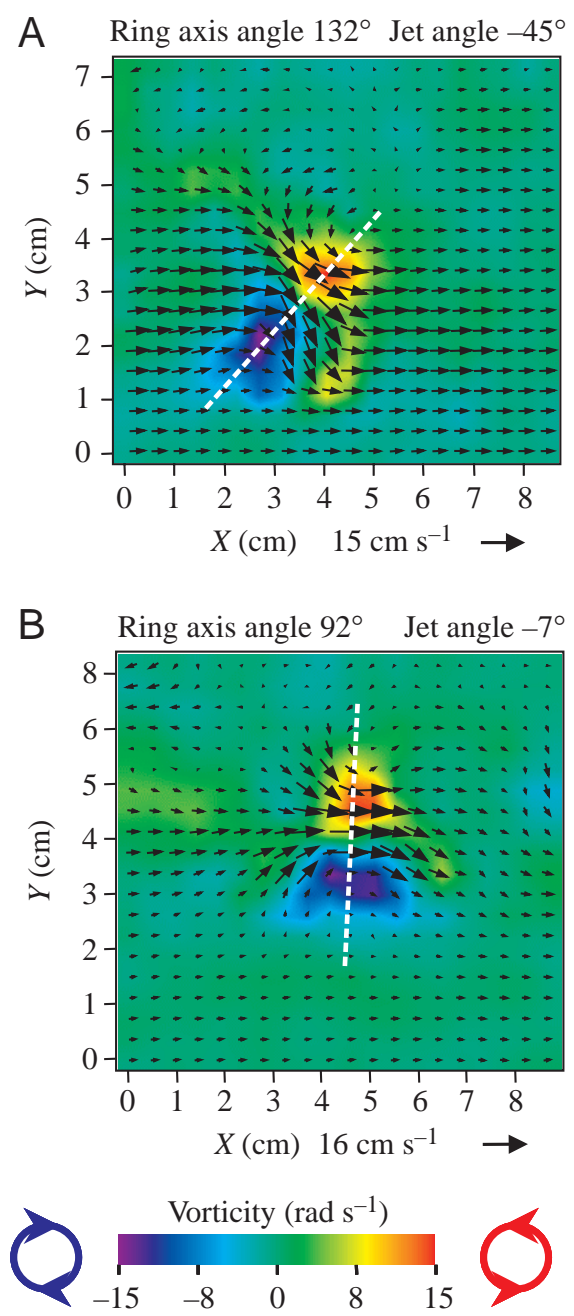

Fig. 6. Representative DPIV analyses of the wake of the tail of Triakis semifasciata while (A) rising and (B) sinking in the water column. Each color plot shows fluid vorticity, with superimposed black velocity vectors representing the results of DPIV calculations as in Fig. 4. During rising behavior, the direction of the jet is similar to that while holding vertical position during horizontal locomotion, although the ring axis angle is inclined more horizontally. In contrast, during sinking behavior, the fluid jet is significantly more horizontal and the ring axis angle is significantly more vertically inclined. To assist in visualizing the flow pattern, a mean horizontal flow of $U=19$ and $U=24 \mathrm{~cm} \mathrm{~s}^{-1}$ was subtracted from each vector for rising and sinking, respectively. The white dashed line indicates the ring axis cycle. 
Table 1. Summary statistics of DPIV variables in Triakis semifasciata and Chiloscyllium punctatum holding position at $1.0 \mathrm{~L} \mathrm{~s}^{-1}$ and during vertical maneuvering in Triakis semifasciata

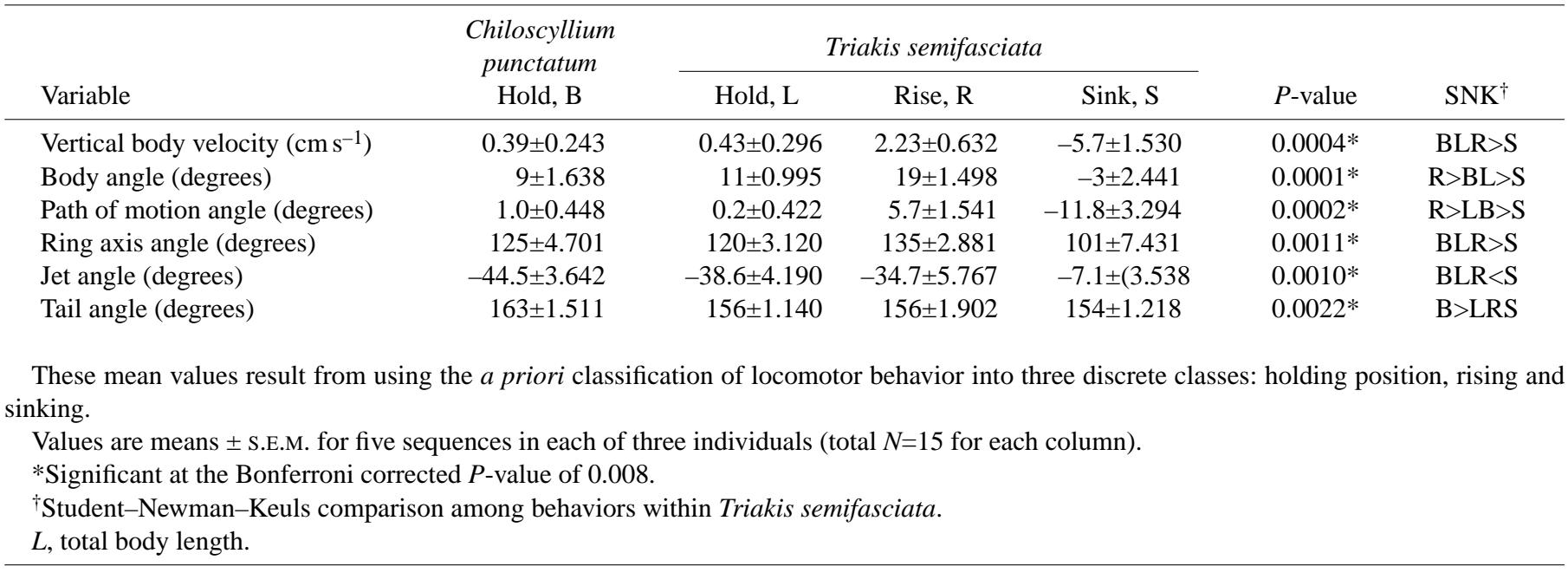

jet angle and ring axis angle. Slopes were first tested for significance and then tested statistically against the slope of the expected relationships based on a priori geometric relationships between body angle, ring axis angle and vortex jet angle. Student's $t$-tests were used to test the significance of the intercepts and slopes between data regression lines and predicted lines according to Zar (1996). The same variables were used in analyses of locomotor behavior, which consisted of a mixed-model two-way analysis of variance (ANOVA) using Type III sums of squares (Hicks, 1982; SAS Institute, 1998). Behavior (rising, holding or sinking) was treated as a fixed main effect and individual as a random main effect; consequently, behavior was tested over the behavior $x$ individual interaction term. If a significant difference was detected by ANOVA, then a post-hoc Student-Newman-Keuls (SNK) multiple-comparisons test was performed. Data were tested for homogeneous variances using the Levene median test $(P<0.05)$ and for normal distribution using the Kolmogorov-Smirnov test $(P<0.05)$. Statistical tests were performed using statistical software (SAS v. 6.12 or SigmaStat v. 2.01) or calculated using Zar (1996).

\section{Results}

Leopard and bamboo sharks swim steadily forward in the flow tank at $1 \mathrm{Ls}^{-1}$ with the body tilted at mean angles of 11 and $9^{\circ}$, respectively, to the flow (Tables 1,2). Although body angle is inclined upwards, swimming trajectory is steadily horizontal as demonstrated by the mean path of motion angles of 0.2 and $1.0^{\circ}$, respectively, to the flow, which are not significantly different from zero. Vertical body velocity is very low when sharks hold position, $0.43 \mathrm{~cm} \mathrm{~s}^{-1}$ for leopard sharks and $0.39 \mathrm{~cm} \mathrm{~s}^{-1}$ for bamboo sharks, reflecting effectively horizontal steady locomotion. During vertical maneuvering, leopard sharks adjust their body angle to a mean of 19 and $-3^{\circ}$ while rising and sinking, respectively, with path of motion angles of 5.7 and $-11.8^{\circ}$ respectively (Tables 1,2 ). Vertical velocity increased to
$2.23 \mathrm{~cm} \mathrm{~s}^{-1}$ during rising and $-5.7 \mathrm{~cm} \mathrm{~s}^{-1}$ during sinking. The heterocercal tail of bamboo sharks is inclined more horizontally $\left(163^{\circ}\right)$ than the tail of leopard sharks $\left(156^{\circ}\right)$.

Analysis of vertical light sheet DPIV images of the wake behind the heterocercal tail of leopard and bamboo sharks reveals slices through discrete vortex rings containing a central high-velocity jet of water (Fig. 4). This vortex ring is shed after each tail beat (Fig. 4) and is linked with the vortex ring formed during the subsequent tail beat. Shark tail vortex rings are inclined significantly to the flow with the plane of the vortex ring relative to the horizontal, reaching a mean of $120^{\circ}$ in leopard sharks and $125^{\circ}$ in bamboo sharks during steady horizontal locomotion (Tables 1,2). Plots of vortex jet angle versus body angle indicate that jet angles were, on average, nearly $30^{\circ}$ below the horizontal during steady horizontal locomotion in both leopard and bamboo sharks (Fig. 5; Table 2). However, mean ring axis angle ranges from

Table 2. Predicted values from linear regressions using mean body angle values shown for Triakis semifasciata and

Chiloscyllium punctatum holding position, and for Triakis semifasciata during vertical maneuvering at $1.0 \mathrm{~L} \mathrm{~s}^{-1}$

\begin{tabular}{lcccc}
\hline & $\begin{array}{c}\text { Chiloscyllium } \\
\text { punctatum }\end{array}$ & \multicolumn{2}{c}{ Triakis semifasciata } \\
\cline { 3 - 5 } Variable & Hold & Hold & Rise & Sink \\
\hline Body angle (degrees) & 10 & 11 & 19 & -3 \\
Path of motion angle (degrees) & 1.0 & -0.4 & 5.8 & -11.3 \\
Ring axis angle (degrees) & 126 & 121 & 131 & 103 \\
Jet angle (degrees) & -43.8 & -28.9 & -37.6 & -13.7 \\
Tail angle (degrees) & 163 & 155 & 155 & 155
\end{tabular}

Values are from five sequences per behavior from each of three individuals per species.

These mean values result from using regression equations for all locomotor behaviors (see Figs 5, 7, 8).

$L$, total body length. 
55 to $160^{\circ}$ during vertical maneuvering in leopard sharks (Fig. 7C).

Leopard sharks do not alter their tail angle with changes in body angle, as shown by the lack of difference in the slope of the regression line from the predicted $180^{\circ}$ linear relationships between tail and body angles (Fig. 7A). Thus, the tail maintains a consistent angular relationship with the body regardless of locomotor behavior. Ring axis angle also retains a consistent relationship to body angle, as shown by the lack
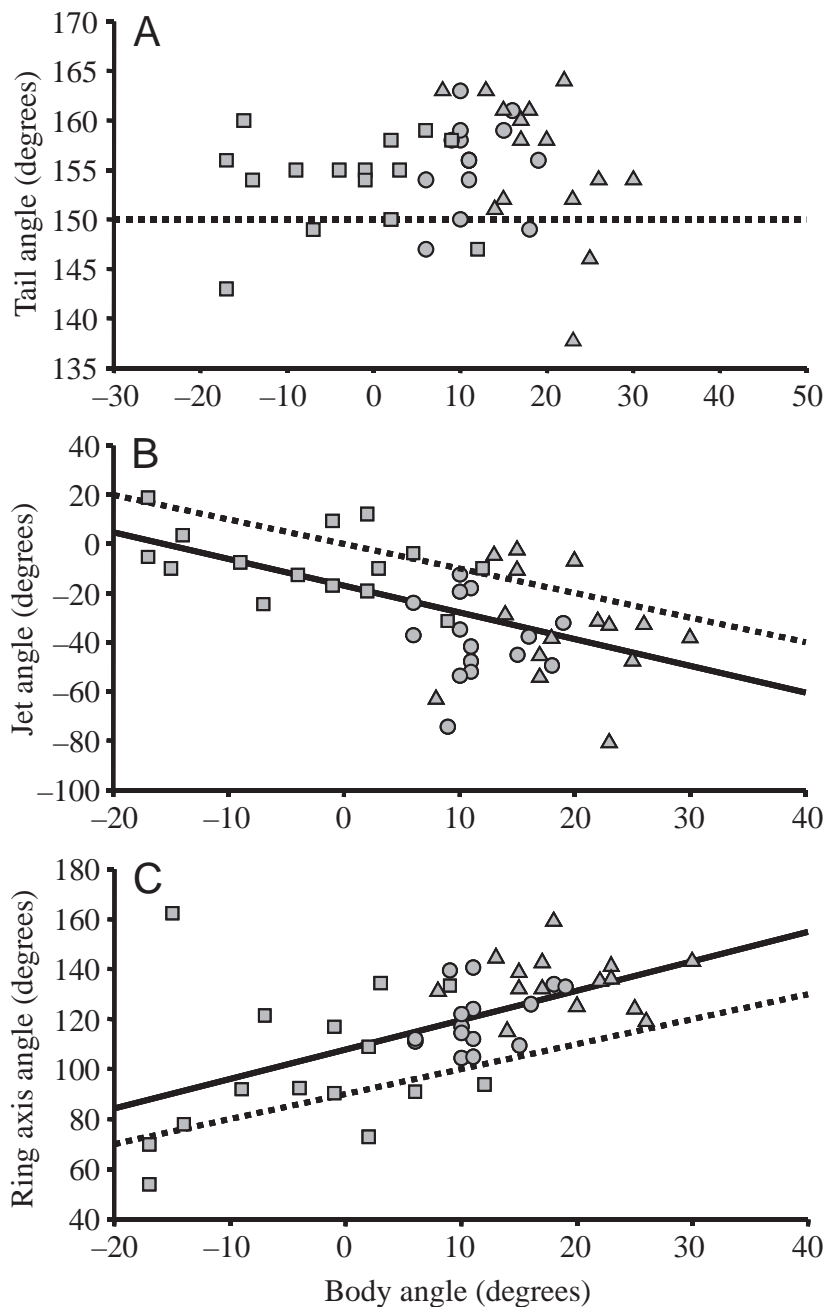

Fig. 7. Plots of body angle versus (A) tail angle, (B) jet angle and (C) ring axis angle in Triakis semifasciata. The solid lines indicate a significant linear regression, while the dotted lines represent the predicted relationships (see text for discussion). The lack of significance of the tail versus body angle regression $(P=0.731$, $\left.r^{2}=0.003\right)$ indicates that the sharks are not altering their tail angle as body angle changes, but instead are maintaining a constant angular relationship regardless of locomotor behavior. Jet angle decreases with increasing body angle $\left(y=-17-1.087 x ; P<0.001, r^{2}=0.312\right)$ at the same rate as the predicted parallel relationship, indicating that the vortex jet is generated at a constant angle to the body regardless of body position. Ring axis angle increases with body angle at the same rate as the predicted perpendicular relationship $(y=107+1.280 x$; $\left.P<0.001, r^{2}=0.401\right)$. Circles, triangles and squares represent holding, rising and sinking, respectively. of significant difference between the slope of the regression line from that of the $90^{\circ}$ perpendicular predicted relationship (Fig. 7C). In fact, ring axis angle averages $18^{\circ}$ greater than the predicted $90^{\circ}$ relationship, indicating that vortex rings are produced at an angle of approximately $108^{\circ}$ to the shark body. Jet angle decreases with increasing body angle at the same rate as to be expected if a parallel relationship were predicted (shown by the lack of a significant difference between the slope of the regression line from that of the $180^{\circ}$ parallel predicted relationship; Fig. 7B).

Leopard sharks do not alter their jet angle with their path of motion angle, as shown by the lack of significant difference between the slopes of the regression line from that of the $180^{\circ}$ parallel predicted relationship (Fig. 8A). The data regression line is approximately $28^{\circ}$ lower than that predicted; therefore, the tail vortex jet is produced at an angle of $152^{\circ}$ to the path of motion followed by the shark. However, a plot of ring axis angle versus jet angle shows a significant departure from the $90^{\circ}$ predicted relationship (Fig. 8B). Jet angle decreases with

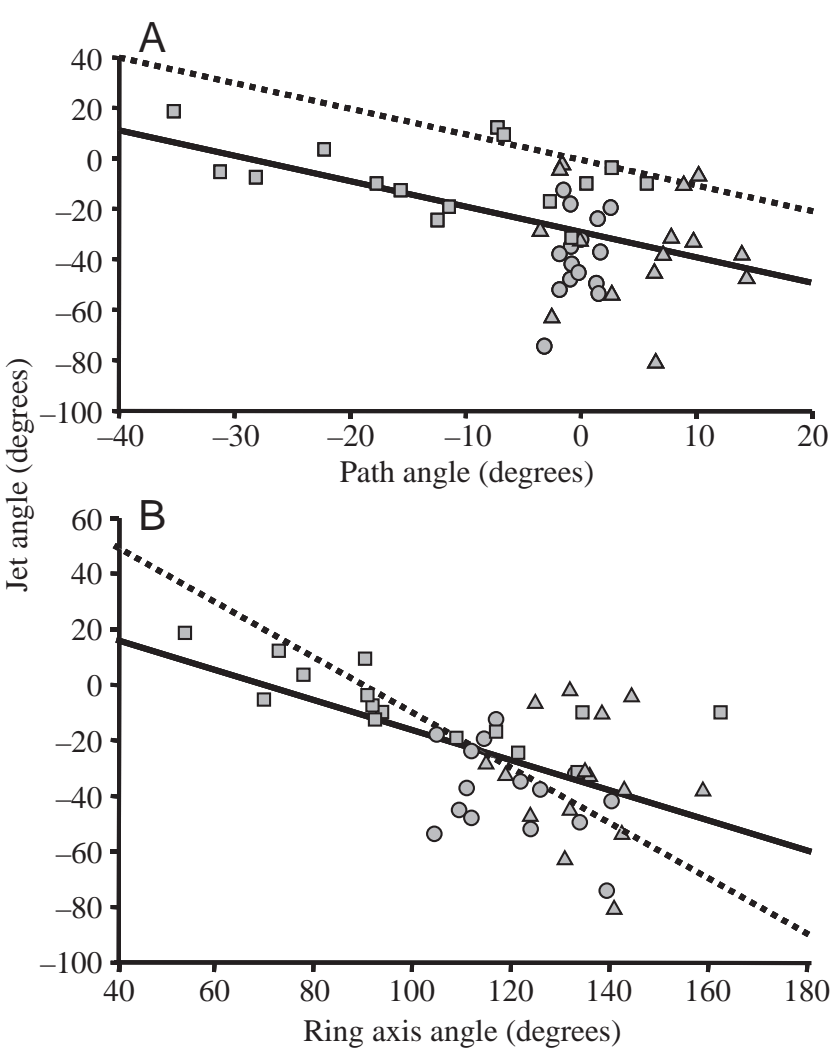

Fig. 8. Plots of jet angle versus (A) path of motion angle and (B) ring axis angle for Triakis semifasciata. The solid lines indicate a significant linear regression, while the dotted lines represent the predicted relationships (see text for discussion). Jet angle has a significant negative correlation with path angle $(y=-29-1.045 x$; $\left.P<0.001, r^{2}=0.257\right)$ and parallels the predicted relationship in which vortex jets are oriented in a direction opposite to the path of motion. Jet angle decreases with increasing ring axis angle at a slower rate than expected assuming a perpendicular relationship $\left(y=34.720-0.519 x ; \quad P<0.001, \quad r^{2}=0.291\right)$. Circles, triangles and squares represent holding, rising and sinking, respectively. 
increasing ring axis angle at a slower rate than to be expected if a perpendicular relationship were to exist. Thus, jet angle remains closer to the horizontal than expected as ring axis angle changes.

\section{Discussion}

Visualization and quantification of the wake in both horizontal and vertical planes of the heterocercal tail of leopard (Triakis semifasciata) and bamboo sharks (Chiloscyllium punctatum) sharks reveal that a vortex ring surrounding a highvelocity jet of water is shed into the wake after each tail beat, generating vortex rings in the wake of the tail. This paper has focused on the vertical light sheet data to quantify the direction of the reaction force to this central jet, which is the force that acts to generate torque around the center of mass of the shark. Although heterocercal tail angle and external morphology differ significantly between leopard and bamboo sharks, the vortex ring jet angle produced is generally similar, -38 and $-44^{\circ}$ respectively (Table 1 ), suggesting that the interspecific differences in heterocercal tail morphology between the two shark species studied here have little effect on basic hydrodynamic tail function. The heterocercal tail of both species generates high-angle reaction forces considerably dorsal to the center of mass.

If the shark tail functions to generate a reaction force that passes through the center of mass, as suggested by Thomson (1976), then vortex jet angles must be equal and opposite to the body angle, even when the body angle is altered during vertical maneuvering. Mean jet angles were nearly $30^{\circ}$ below the horizontal in leopard and bamboo shark tails during steady horizontal locomotion, as revealed by plotting vortex jet angle versus body angle (Fig. 5). The reaction force from the tail vortex rings of both leopard and bamboo sharks must be directed anterodorsally, as predicted by the classical model of heterocercal tail function in sharks (Alexander, 1965; Ferry and Lauder, 1996), since a mean positive $11^{\circ}$ body tilt is adopted during steady horizontal swimming (Fig. 9; holding). Furthermore, leopard sharks maintain a consistent relationship between jet and body angle during unsteady maneuvering locomotion, as indicated by the linear relationship paralleling the $180^{\circ}$ predicted line. The direction of vortex ring jets is not altered by leopard sharks while maneuvering vertically and, thus, the heterocercal tail generates a jet force that is constant in direction relative to the longitudinal body axis. In notable contrast to this result, white sturgeon Acipenser transmontanus are capable of actively altering the angle of jet flow produced by the heterocercal tail by up to $10^{\circ}$, as shown by Liao and Lauder (2000). The basis for this difference in ability between sturgeon and the two shark species studied here to modulate vortex jet direction is unknown, but might reflect their differing abilities to recruit dorsal and ventral myotomal musculature differentially to change tail shape and flexibility and, hence, to alter the direction of thrust from the tail.

Vortex rings in the wake of the shark tail are inclined relative to the flow with the plane of the vortex ring averaging $120^{\circ}$ during steady horizontal locomotion and ranging from 55 to $160^{\circ}$ while maneuvering. Shark tail vortex rings are inclined significantly more towards the horizontal compared with the more nearly vertical vortex rings produced by the homocercal tail of bluegill sunfish Lepomis macrochirus (Lauder, 2000) and mackerel Scomber japonicus (Nauen and Lauder, 2002). Indeed, compared with those of a ray-finned fish with a heterocercal tail (the sturgeon), shark vortex rings are inclined approximately $15^{\circ}$ more towards the horizontal (Liao and Lauder, 2000) during steady horizontal locomotion. The heterocercal tail of sturgeon generates reaction forces directed through the center of mass of the body, while the heterocercal tail of sharks results in reaction forces directed dorsal to the center of mass (Fig. 9).

Why heterocercal tails produce vortex rings that are more inclined relative to body angle than homocercal tails has yet to be investigated. It may simply be an effect of the inclined posterior edge of the caudal fin. If the vortex ring is shed simultaneously from the dorsal and ventral lobes, then it would tend to maintain a tilted axis as it rolls off the edge of the dorsal and ventral fin lobes into the wake. As sharks rise in the water column, the trailing edge of the tail is more horizontally oriented, generating vortex rings that tend to be inclined more horizontally (Fig. 6A; Tables 1,2). As sharks sink in the water column, the posterior edge of their tail is more vertical, generating vortex rings that have a more vertical axis (Fig. 6B). This, together with the constant angle of the tail during all behaviors (holding, rising, sinking), is consistent with the idea that tilted rings are an effect of tail trailing edge shape and movement.

Comparative studies show, however, that vortex ring angle is not necessarily directly related to the morphological angle formed by the trailing edge of the tail; the kinematics of the tail also plays a major role in determining vortex ring orientation. For example, in sturgeon (Liao and Lauder, 2000), vortex rings shed during steady locomotion are more vertically oriented than would be predicted from trailing edge angle as a result of the complex three-dimensional motion of the tail tips (Lauder, 2000). In homocercal tails, which have a primarily vertical trailing edge, shed vortex rings may be inclined significantly to the vertical or have non-horizontal jet flow as a result of asymmetrical movement of the dorsal and ventral tail tips (Lauder, 2000; Nauen and Lauder, 2002).

Differences between shark and sturgeon tail function may be due to significant differences in kinematics. Lauder (2000) described sturgeon tail kinematics and noted that the dorsal and ventral lobes are often significantly out of phase with each other and that oscillation of surface elements of sturgeon tails occurs around the vertical plane. During locomotion, sturgeon tails show remarkable flexibility, and portions of the dorsal lobe move in the opposite direction to the ventral tail lobe for much of the tailbeat cycle. This is in sharp contrast to the kinematic pattern described for shark tails by Ferry and Lauder (1996). Shark tails possess considerable internal stiffness compared with sturgeon tails and move at an inclined angle to the horizontal much in the manner proposed by the 
classical model (Fig. 1A). Phase differences among parts of the tail in sharks never approach those seen for sturgeon (Lauder, 2000). Differences in tail kinematics between sharks and sturgeon thus appear to correlate with observed hydrodynamic differences in vortex jet angle relative to the center of mass.

Although our hydrodynamic data support the classical model of heterocercal tail function in sharks, our previous analyses of the hydrodynamic function of the pectoral fins in sharks contradicts the classical view that the pectoral fins generate lift forces during steady horizontal locomotion (Wilga and Lauder, 2000, 2001). Three-dimensional kinematic analyses of the pectoral fins of leopard and bamboo sharks show that these fins are held in a concave-down orientation at a mean chord angle of $-5^{\circ}$ to the flow. Thus, leopard shark pectoral fins are not held at a positive angle of attack to the flow during steady horizontal locomotion and should not be expected to generate lift. In addition, DPIV analyses of the pectoral fin wake reveal that the pectoral fins generate no lift forces during steady horizontal swimming (Wilga and Lauder, 2000, 2001).

Combining the hydrodynamic and kinematic data on pectoral fin, body posture and caudal fin function in leopard and bamboo sharks during steady horizontal swimming with that for leopard sharks during vertical maneuvering suggests a new force balance for shark locomotion (Fig. 9). Vertical forces (F) generated by swimming sharks are separated into the head and branchial region $\left(F_{\text {head }}\right)$ and pectoral fins $\left(F_{\text {pectoral }}\right)$, which are anterior to the center of mass, and the body ( $\left.F_{\text {body }}\right)$ and tail $\left(F_{\text {tail }}\right)$, which are posterior to the center of mass. The body weight $\left(F_{\text {weight }}\right)$ of the shark acts at the center of mass. The vortex jet force $\left(F_{\text {jet }}\right)$ produced by the tail is equivalent and opposite in direction to the reaction force on the tail $\left(F_{\text {reaction}}\right)$. Our new force balance proposes that the torque produced by the heterocercal tail

Fig. 9. Schematic diagram of the force balance on Triakis semifasciata swimming at $1.0 \mathrm{~L} \mathrm{~s}^{-1}$, where $L$ is total body length, while holding position (also representative of Chiloscyllium punctatum), rising and sinking in the water column. The yellow circle represents the center of mass, and vectors indicate forces $F$ exerted by the fish on the fluid. Lift forces are generated by the ventral body surface, both anterior and posterior to the center of mass. The jet produced by the beating of the tail maintains a constant angle relative to body angle and path angle and results in an anterodorsally directed reaction force oriented dorsal to the center of mass during all three behaviors, supporting the classical model. Data on pectoral fin function are from Wilga and Lauder (2000, 2001). Tail vortex jet angles are predicted mean values from Table 2 .

SINKING during steady horizontal swimming by leopard sharks is balanced by the torque generated by the relatively large positive body angle to the flow, which generates lift forces both fore and aft of the center of mass, and not by the pectoral fins (Fig. 9).

Although our hydrodynamic and kinematic data on shark pectoral fins indicate that the pectoral fins generate no lift during steady horizontal locomotion, the pectoral fins of leopard sharks are used actively to initiate rising and sinking maneuvers, during which positive and negative lift forces, respectively, are actively generated by the pectoral fins (Wilga and Lauder, 2000, 2001). During rising, the pectoral fins shed a vortex that generates positive lift and acts to increase the body angle of the shark, which increases the lift generated by the tilted body. During sinking, the pectoral fins generate a vortex with negative lift that acts to tilt the body angle to a more negative angle relative to the flow.

The experimental hydrodynamic and three-dimensional kinematic analyses of shark locomotion show that body forces are balanced in an unexpected manner. Although the classical model of heterocercal tail function in sharks is supported, the locomotor roles of the pectoral fins and body posture have not
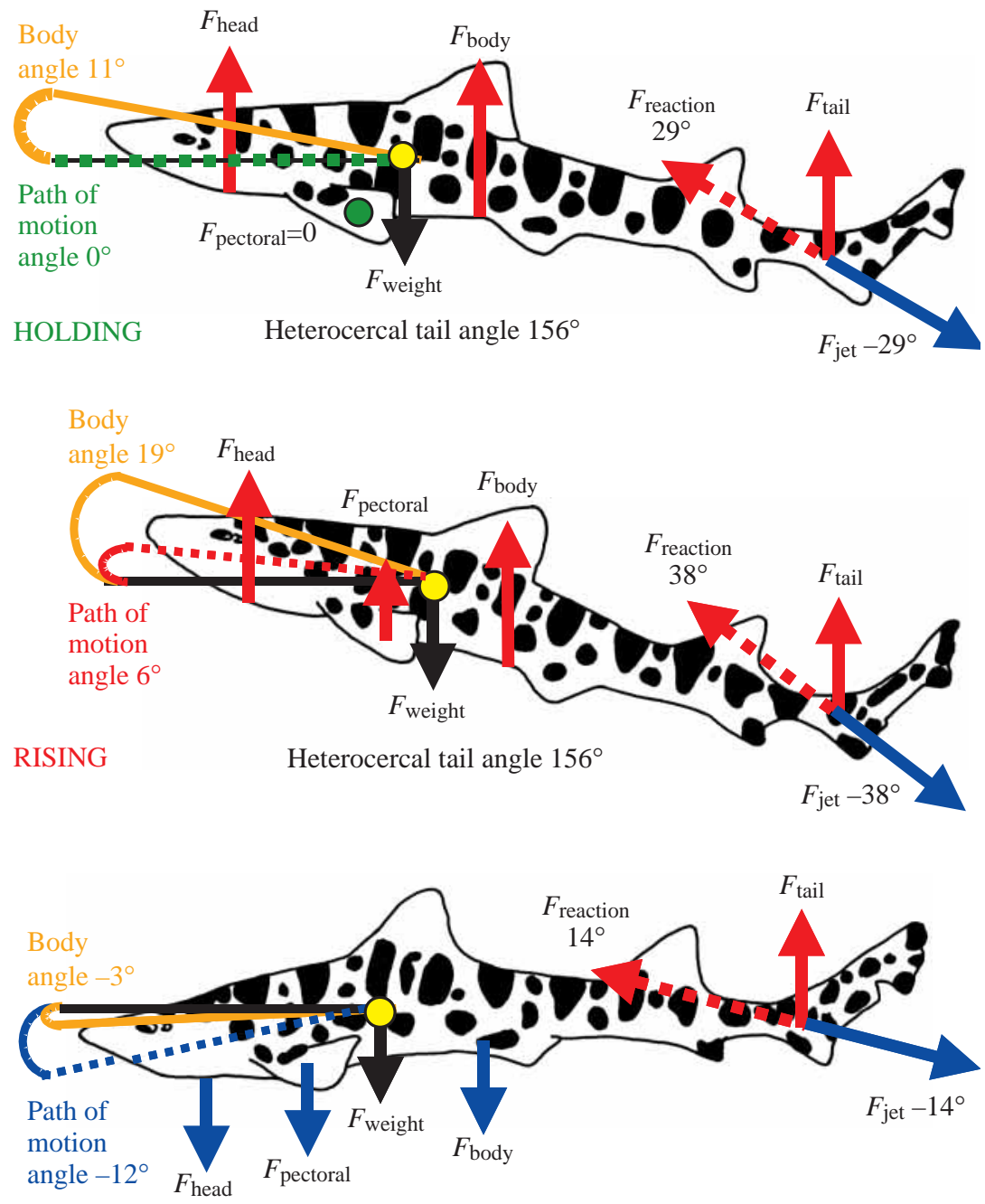

Heterocercal tail angle $154^{\circ}$ 


\section{C. D. Wilga and G. V. Lauder}

been previously recognized. The advent of experimental hydrodynamic techniques allows long-standing hypotheses of fin function in fishes to be tested, and such approaches will play a key role in elucidating the functional significance of variation in fin morphology among the considerable diversity of shark species and locomotor modes.

We thank Jocelyne Dolce and Laura Farrell for technical assistance and Jimmy Liao, Eliot Drucker, Jennifer Nauen and Tonia Hsieh for helpful discussions. We thank Emily Carrington, Jimmy Liao and two anonymous reviewers for helpful comments on the manuscript. Support was provided by National Science Foundation grants to C.D.W. (DBI 9707846) and G.V.L. (IBN 98-07012) and from the University of Rhode Island to C.D.W.

\section{References}

Affleck, R. J. (1950). Some points in the function, development and evolution of the tail in fishes. Proc. Zool. Soc., Lond. 120, 349-368.

Alexander, R. McN. (1965). The lift produced by the heterocercal tails of Selachii. J. Exp. Biol. 43, 131-138.

Drucker, E. G. and Lauder, G. V. (1999). Locomotor forces on a swimming fish: three-dimensional vortex wake dynamics quantified using digital particle image velocimetry. J. Exp. Biol. 202, 2393-2412.

Drucker, E. G. and Lauder, G. V. (2000). A hydrodynamic analysis of fish swimming speed: wake structure and locomotor force in slow and fast labriform swimmers. J. Exp. Biol. 203, 2379-2393.

Drucker, E. G. and Lauder, G. V. (2001). Locomotor function of the dorsal fin in teleost fishes: experimental analysis of wake forces in sunfish. J. Exp. Biol. 204, 2943-2958.

Ferry, L. A. and Lauder, G. V. (1996). Heterocercal tail function in leopard sharks: a three-dimensional kinematic analysis of two models. J. Exp. Biol. 199, 2253-2268.

Gibb, A., Jayne, B. C. and Lauder, G. V. (1994). Kinematics of pectoral fin locomotion in the bluegill sunfish Lepomis macrochirus. J. Exp. Biol. 189, 133-161.
Grove, A. J. and Newell, G. E. (1936). A mechanical investigation into the effectual action of the caudal fin of some aquatic chordates. Ann. Mag. Nat. Hist. 17, 280-290.

Hicks, C. R. (1982). Fundamental Concepts in the Design of Experiments. Orlando, FL, USA: Saunders College Publishing.

Jayne, B. C. and Lauder, G. V. (1995). Speed effects on midline kinematics during steady undulatory swimming of largemouth bass, Micropterus salmoides. J. Exp. Biol. 198, 585-602.

Lauder, G. V. (2000). Function of the caudal fin during locomotion in fishes: kinematics, flow visualization and evolutionary patterns. Am. Zool. 40, $101-122$

Liao, J. and Lauder, G. V. (2000). Function of the heterocercal tail in white sturgeon: flow visualization during steady swimming and vertical maneuvering. J. Exp. Biol. 203, 3585-3594.

Nauen, J. C. and Lauder, G. V. (2001). Locomotion in scombrid fishes: visualization of flow around the caudal peduncle and finlets of the chub mackerel Scomber japonicus. J. Exp. Biol. 204, 2251-2263.

Nauen, J. C. and Lauder, G. V. (2002). Hydrodynamics of caudal fin locomotion by chub mackerel Scomber japonicus. J. Exp. Biol. 205, 1709-1723.

Raffel, M., Willert, C. and Kompenhans, J. (1998). Particle Image Velocimetry: A Practical Guide. Heidelberg: Springer-Verlag.

SAS Institute (1998). SAS/STAT System for Personal Computers, version 6.12. Cary, NC, USA: SAS Institute.

Simons, J. R. (1970). The direction of the thrust produced by the heterocercal tails of two dissimilar elasmobranchs: the Port Jackson shark, Heterodontus portusjacksoni (Meyer) and the piked dogfish, Squalus megalops (Macleay). J. Exp. Biol. 52, 95-107.

Thomson, K. S. (1976). On the heterocercal tail in sharks. Paleobiology 2 , 19-38.

Thomson, K. S. and Simanek, D. E. (1977). Body form and locomotion in sharks. Am. Zool. 17, 343-354.

Wilga, C. D. and Lauder, G. V. (1999). Locomotion in sturgeon: function of the pectoral fins. J. Exp. Biol. 202, 2413-2432.

Wilga, C. D. and Lauder, G. V. (2000). Three-dimensional kinematics and wake structure of the pectoral fins during locomotion in leopard sharks, Triakis semifasciata. J. Exp. Biol. 203, 2261-2278.

Wilga, C. D. and Lauder, G. V. (2001). Functional morphology of the pectoral fins in bamboo sharks, Chiloscyllium plagiosum: benthic versus pelagic station holding. J. Morphol. 249, 195-209.

Zar, J. H. (1996). Biostatistical Analysis. Upper Saddle River, NJ: Prentice Hall. 